\title{
Research on the Ground Penetrating Radar Clutter Suppression Technology Based on PCA Method
}

\author{
DeMei YANG ${ }^{1, a}$, Yao QIN ${ }^{2, b}$, Xiaozhen $\operatorname{Ren}^{3, c}$ \\ ${ }^{1}$ College of Information Science and Engineering, Henan University of Technology, Zhengzhou, \\ 450000, China \\ ${ }^{2}$ College of Information Science and Engineering, Henan University of Technology, Zhengzhou, \\ 450000, China \\ ${ }^{3}$ College of Information Science and Engineering, Henan University of Technology, Zhengzhou, \\ 450000, China
}

aemail: ymei93@163.com, bemail:eqinyao@163.com, email:yxf236@163.com

Keywords: Ground Penetrating Radar; Principal Component Analysis; clutter suppression

\begin{abstract}
The reduction of background clutter signal from Ground Penetrating Radar (GPR) experiments is an area of active research. The weak reflection signal obtained from subsurface targets is usually blurred by such strong clutter. Therefore, clutter suppression is the key step in ground penetrating radar signal processing. In this paper, Principal Component Analysis (PCA) algorithm is used to suppress GPR clutter. First, mean filter method is used to remove the direct wave and part of noise signal in the reflection wave, and then the processed observing data are divided into target space and clutter space. After that, signals in target space are decomposed into independent components by dimension reduction. Finally, removing small components can get the purposes of outstanding effective target and clutter suppression. By dealing with GPR experiment data, it shows that the PCA algorithm based on mean filter to suppress clutter is effectiveness and stability.
\end{abstract}

\section{Introduction}

In recent years, Ground Penetrating Radar (GPR) technology is widely used in the subsurface detection. Experiment GPR data are mixed signals which are generated by direct wave, target signal, random noise, and interference signal formed by false target [1]. The problem of clutter in observation signal is always an important aspect of impact GPR target detection performance. If the target depth is not very deep, the clutter signal can overlaps with the target echo signal, the target identification and explanation can be affected. Therefore, effectively suppress clutter becomes an important problem in signal processing of GPR, in order to get a more accurate and effective data.

Therefore many methods are put forward, such as mean filter method, the wavelet method, the ICA method, etc. These methods have achieved certain effect some situations. But there are also some shortcomings. In this paper, the Principal Component Analysis (PCA) method through the data compression and dimension reduction, can effectively reduce the redundant information in the data, and extract the main components of the target, achieve the purpose of clutter suppression and outstanding target feature.

PCA as a kind of analysis method on the basis of the optimal principle, has a long history. In 1901, Pearson introduced the transform into the field of biology. In 1933 Hoteling adopted this idea for psychological research and got further development, and then Loeve summarized and generalized. The PCA algorithm is an optimal transformation of the minimum mean square sense, reduced dimension in the case of nondestructive or little loss of information, aiming at GPR signal, the PCA method can dimension reduction based on retain the main target components, decompose signal into independent components, remove the clutter in the signal, outstanding the effective target, easy to extract target feature, in the link radar target recognition system on the expression of the target property is essential.

In performance, in order to match actual system performance requirements, the number of 
principal components of PCA can be freely increased or decreased [2]. After dimension reduction, we can retain the greatest degree of feature information, and achieve the recognition of radar target feature extraction based on ensure the recognition performance. In economy, the ideal processing effect, the computational overhead in subsequent phases will be greatly reduced. So, PCA clutter suppression method can use less computational cost to achieve more ideal performance.

\section{Basic principle of PCA}

PCA is a data analysis method based on second order statistics, which use a new set of independent variables replace the original correlated variables, these new variables can reflect the original complex variables information as much as possible [3].

PCA performed mathematical decomposition of the original data that reduce the data dimensions of a set of highly complex B-scan data to a smaller number of scores and principal components that effectively carries all the important information of the signal [4]. Classification of data can be easily done by choosing different combinations of principal components to build a new coordinate system. After using the mean filter to remove the clutter, dimension reduction which attempts to detect the reduced intrinsic dimension in the high-dimensional feature space is a necessary step for remove the correlation [4].

PCA is one of the most popular linear dimension methods that can extract useful features through the original feature vector to a small subspace projection, find the main elements in the data. This method is optimal in terms of reconstruction. It get the purpose of reduction dimension and redundancy, also outstanding the implied feature of the original data [5].

Below, let us give a brief introduction about its formulation.

Assume given a data set of GPR in a m-dimensional B-scan, the objective of PCA is to find a transformation matrix $\mathrm{Z}$ to map $\mathrm{X}$ into $\mathrm{R}$ in a new $\mathrm{n}$-dimensional real space, the calculation steps are as follows:

Step1: Through the mean filter, remove the clutter.

The principle of mean filter method is each element of each line of the received B-scan data $G_{m \times n}$ minus the mean of the line, in order to removal of clutter simply [6].

$$
\mathrm{X}_{\mathrm{ij}}=G_{i j}-\frac{1}{\mathrm{n}} \sum_{j=1}^{n} G_{i j}
$$

Step2: In order to obtain an optimized transformation matrix Z, we need to construct a sample array $\mathrm{Z}$ following standardized transformation:

$$
\mathrm{Z}_{\mathrm{ij}}=\frac{x_{i j}-\overline{x_{j}}}{S_{j}}, i=1,2, \ldots, m ; j=1,2, \ldots, n
$$

where $\overline{\mathrm{x}_{\mathrm{j}}}=\frac{\sum_{i=1}^{m} x_{i j}}{m}$, and $\mathrm{S}_{\mathrm{j}}=\sqrt{\frac{\sum_{i=1}^{m}\left(x_{i j}-\overline{x_{j}}\right)^{2}}{m-1}}$.

Step3:Demand the matrix of correlation coefficient for standardized array Z:

$$
\mathrm{R}=\frac{1}{m-1} Z^{T} Z=\left(r_{i j}\right)_{\mathrm{n} \times n}
$$

Step4: Solution the characteristic equation $\left|\mathrm{R}-\lambda \mathrm{I}_{\mathrm{n}}\right|=0$ for $\mathrm{R}$, get $\mathrm{n}$ characteristic values $\lambda_{1}, \lambda_{2}, \ldots, \lambda_{n}$, corresponding feature vectors $p_{1}, p_{2}, \ldots, p_{n}$.

Step5: Characteristic values in descending order, and adjust their corresponding feature vectors.

Step6: Through the Schmidt orthogonal method units orthogonal the feature vector, get $a_{1}, a_{2}, \ldots, a_{n}$. 
Step7: Calculate the cumulative contribution rate $\mathrm{B}=\frac{\sum_{j=1}^{k} \lambda_{j}}{\sum_{\mathrm{j}=1}^{n} \lambda_{j}}$, according to the given extraction efficiency $\eta$ ( generally take a higher percentage, $80 \%-90 \%$ ), if the contribution rate $B \geqq \eta$, determine the value of $\mathrm{k}$, then extracted $\mathrm{k}$ principal components $a_{1}, a_{2}, \ldots, a_{k}$. At this time, not only to achieve the purpose of dimensional reduction, with little loss of information.

Step8. Calculate the projection of the standardized data $\mathrm{Z}$ in the feature vector, that is transform the standardized variables as the main components $U=Z \cdot a$, which $a=\left(a_{1}, a_{2}, \ldots, a_{k}\right)$.

$U_{1}$ is the first principle component, $U_{2}$ is the second principle component, and $U_{k}$ is the kth principle component. The principle components are ordered in a trend of decreasing the variance, so the trend of principle components according to the variance is $U_{1}>U_{2}>\cdots>U_{k}$. It is evident that principle components are uncorrelated with each other.

\section{Experiment data processing}

Figure 1 is a GPR experiment data profile. The horizontal and vertical coordinates are trace and sample point respectively. Figure 2 is the mean filter result. The principle of mean filter is that each element of each line in the received two-dimensional matrix of B-scan data minus the mean of the line. Mean filter method is used to remove direct wave. Figure 3 is the processing result of the PCA. Figure 3 shows that PCA method not only can get rid of multiple reflected waves and random noises, but also can position the targets. It is very helpful to do further research as target recognition and parameter estimation.

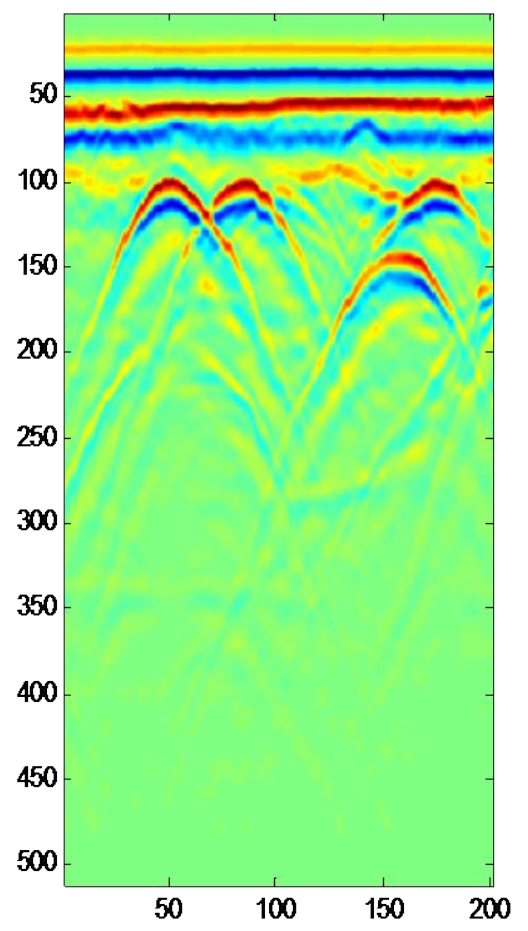

Fig.1 GPR experiment data profile

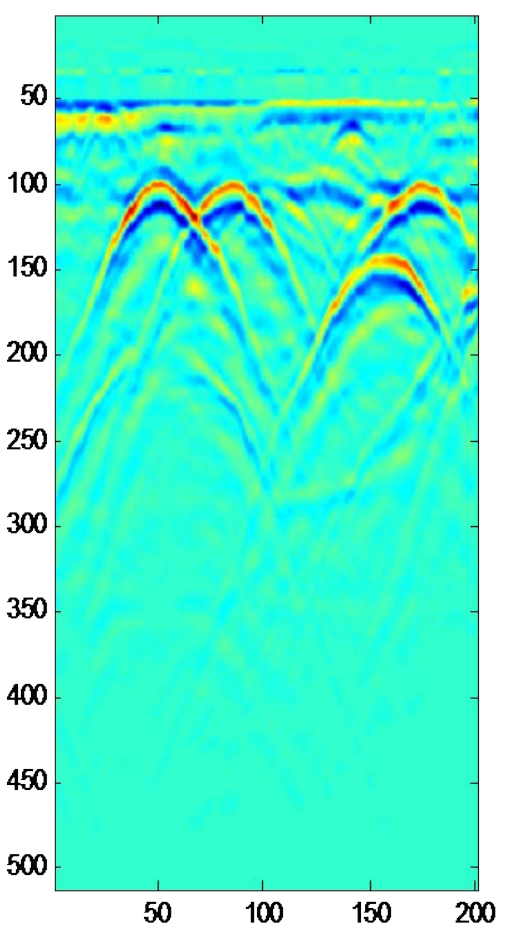

Fig.2 The mean filter result

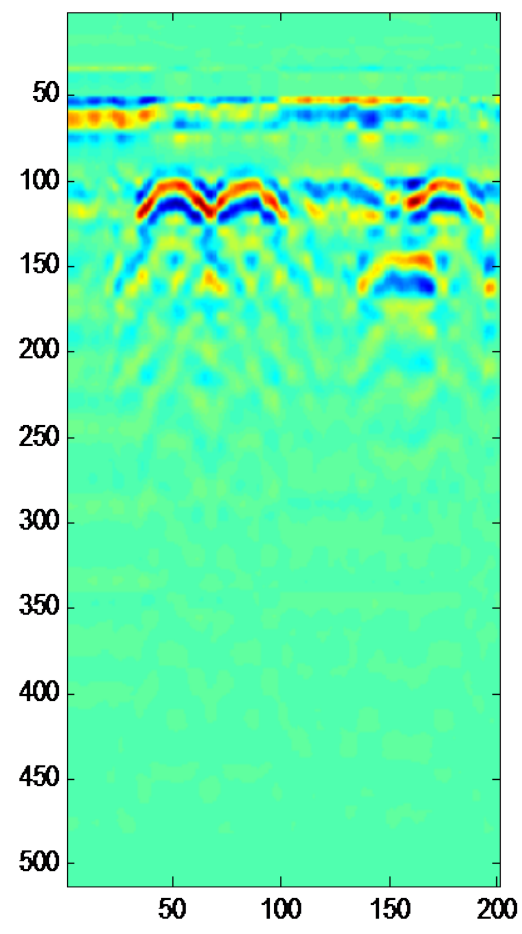

Fig.3 The processing result by PCA 


\section{Conclusion}

The reflection signal obtained from subsurface targets is usually blurred by strong clutter signal, the target identification and explanation can be affected. In this paper, PCA algorithm though reduce dimension, remove the correlation among the the original vector, retain the most important part of signal, and omit the later information. The method can achieve the effect of data compression, and the greatest degree to retain the information of the original data, so as to outstanding the implied features in the original data. The results of the experiment data processing shows that the PCA algorithm can effectively the suppress clutter and outstanding target feature, so as to the identify and explain target. This method improve the target detection performance of GPR.

PCA algorithm does not consider the higher-order statistics characteristic of signal data. It also has some useful information of targets. In the future, we will focus on the research of higher-order statistics of PCA, so as to improve effect of clutter suppression and the accuracy of target positioning.

\section{Acknowledgement}

The authors would like to thank for the support by National Natural Science of China Foundation under Grant 61201389, 61201390, and 11201120. The authors also thank for the support by Provincial Colleges and Universities on Basic Research Fund of Henan Technology University under Grant 2014YWQQ11.

\section{References}

[1] Anxing Zhao, Yansheng Jiang, Wenbing Wang. A primary study of independent component analysis for GPR signal processing [J]. Coal Geology \& Exploration. 2005: 33 (6): 64-67.

[2] Shan Ouyang. A space-time adaptive processing method for airborne radars by using the principal component analysis [J]. Journal of Guilin institute of electronic technology. 2000: 20 (4): 42-46.

[3] Jolliffe I T. Principal Component Analysis [M]. 2nd Edition, New York: Springer. 2002. [2].

[4] Wang Li-Peng, Yuan Zhan-Ting, Chen Xu-Hui, Zhou Zhi-Fang. PCA and KPCA for predicting membrane protein types, WRI Global Congress on Intelligent Systems. 2009 (2): 175-178.

[5] Liang Shengjie, Zhang Zhihua, Cui Lilin. Comparison between PCA and KPCA method in dimensional reduction of mechanical noise data [J]. China Mechanical Engineering. 2011: 22 (1): 80-83.

[6] Yongbin Yan, Image identification and disposal of Ground Penetrating Radar [D]. Beijing: Beijing University of Chemical Technology. 2008: 20. 